\title{
Diferenciais de mortalidade por causas nas faixas etárias limítrofes de idosos
}

\author{
Mortality differentials by cause in extreme age groups of elderly
}

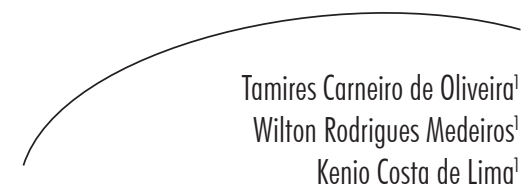

Resumo

Objetivo: Identificar e comparar a escala de prioridades na saúde de idosos mais jovens (60 a 69 anos de idade) e longevos (80 anos ou mais), segundo causas de mortalidade no Rio Grande do Norte, no período de 2001 a 2011. Métodos: Estudo ecológico, cujos dados foram obtidos a partir do Sistema de Informações sobre Mortalidade (SIM) e medidos através da Mortalidade Proporcional (MP). Resultados: As doenças cardiovasculares são a principal causa de morte para os dois grupos, com MP de mais de 30\%. Já o segundo capítulo mais importante se distingue entre os dois grupos, correspondendo às neoplasias $(22,9 \%)$ para os mais jovens, que ocupa a quarta posição entre os longevos $(10,1 \%)$. As causas mal definidas são a segunda mais prevalente para estes $(17,3 \%)$ e a quarta entre os mais jovens $(9,18 \%)$. Há expressivas diferenças em relação às doenças respiratórias, do aparelho digestivo e causas externas. Sobre a primeira, os mais jovens $(12,8 \%)$ têm MP duas vezes maior que os longevos $(6,2 \%)$, enquanto estes possuem MP cerca de duas e três vezes maior para as duas últimas, respectivamente. Conclusão: O estudo indica a heterogeneidade da população idosa, produzindo demandas distintas para as ações em saúde. As doenças cardiovasculares representam a principal causa de óbito para os dois grupos, porém as neoplasias, doenças do aparelho digestivo e causas externas obtiveram maior valor de MP entre os idosos mais jovens, ao passo que as mal definidas e do aparelho respiratório são mais importantes para os longevos.

\section{Abstract}

Objective: To identify and compare the scale of priorities in the health of young-old ( 60 to 69 years old) and oldest-old (older than 80 years) individuals based on the cause of mortality in Rio Grande do Norte state, Brazil, from 2001 to 2011. Methods: For this ecological study, data were obtained from the Brazilian Mortality Information System (SIM) and measured by Proportional Mortality (PM). Results: Cardiovascular diseases are the leading cause of death for both groups, with a PM of over 30\%. The second most common cause among young-old individuals is neoplasm (22.9\%), which only

Palavras-chave: Saúde do Idoso. Mortalidade. Causas de Morte. Idoso de 80 Anos ou mais 
ranks as the fourth most common cause among the oldest-old (10.1\%). Ill-defined causes are the second most prevalent cause among the oldest-old $(17.3 \%)$ and the fourth most prevalent cause among young-old individuals (9.18\%). Significant differences were recorded for respiratory diseases, the digestive tract and external causes. In the case of the former, young-old individuals (12.8\%) exhibited PM that was twice that of the oldest-old $(6.2 \%)$.They also showed values two and three times higher for the latter two causes, respectively. Conclusion: This study indicates the heterogeneity of the elderly, producing distinct demands for public health. Cardiovascular diseases are the leading cause of death in both groups. Neoplasms, digestive diseases and external causes showed the highest amount of PM among young-old individuals, whereas ill-defined and respiratory causes were more significant among oldest-old individuals.
Key words: Health of the Elderly. Mortality. Cause of Death. Aged 80 and over.

\section{INTRODUÇÃO}

Em meio às medidas de condições de vida, as causas de óbitos são consideradas umas das informações de maior relevância para o conhecimento da situação de saúde de uma população, subsidiando o planejamento e a administração de programas. ${ }^{1}$ A causa básica revela a doença inicial da sequência que levou ao falecimento, imaginando-se que o óbito não teria ocorrido na sua ausência e, portanto, ela deve orientar as ações em saúde no sentido de sua prevenção. ${ }^{2}$

A análise da causa básica revela que o padrão de mortalidade brasileiro tem sofrido complexas mudanças, que tendem a se assemelhar ao perfil de países desenvolvidos. ${ }^{3}$ Tais mudanças advêm no sentido de substituir gradativamente as pandemias relacionadas às doenças infecciosas por doenças degenerativas e agravos produzidos pelo homem, sendo esse processo intitulado por Omran ${ }^{4}$ como"transição epidemiológica". O mesmo vem ocorrendo concomitantemente com a transição demográfica, associada ao incremento do número de indivíduos idosos na população, que detêm a maior carga de mortalidade, representando $62,56 \%$ dos óbitos totais em relação ao ano de $2011 .{ }^{5}$

O envelhecimento populacional resulta em mais incapacidades e maior carga de doenças crônico-degenerativas e, associado aos fatores socioeconômicos e de saúde pública, corrobora as mudanças no perfil de morbi-mortalidade da população. Nessa perspectiva, estudos acerca da faixa etária idosa se mostram essenciais para a compreensão do padrão de mortalidade e, por conseguinte, da situação de saúde. Para tanto, faz-se mister considerar o fenômeno do envelhecimento populacional, que ocorre de forma acelerada no Brasil e acompanha o aumento da longevidade dos indivíduos, expresso pelos ganhos na esperança de vida ao nascer, sendo os idosos longevos (80 anos ou mais de idade) a parcela da população que cresce mais rapidamente. ${ }^{6-8}$

Dado que a população idosa apresenta características morfo-fisiológicas, psicológicas e socioeconômicas diferenciadas de outros indivíduos, inclusive em relação aos idosos entre si, a maior longevidade traz uma série de implicações sociais, culturais e epidemiológicas. ${ }^{8}$ Além da longevidade, que caracteriza a grande amplitude da faixa etária geriátrica, a variabilidade no comportamento do declínio das funções entre os indivíduos ao longo do processo de envelhecimento contribui para uma situação de saúde substantivamente heterogênea do grupo de idosos, constituindo um grande desafio para gestores e pesquisadores do sistema de saúde. ${ }^{9}$ Desta forma, os mesmos devem planejar e aplicar ações que estejam em consonância com essa variabilidade, bem como considerar o impacto da população idosa sobre o perfil epidemiológico geral. 
Nesse sentido, o presente trabalho se propôs a fornecer subsídios para o planejamento de políticas públicas que atendam às novas demandas populacionais, contribuindo com a orientação da aplicação de medidas preventivas específicas e de recursos de saúde com base no perfil epidemiológico, tornando mais efetivos os programas, ações e estratégias em saúde.

Para tanto, o estudo teve como objetivo analisar o perfil de mortalidade da população de idosos no Rio Grande do Norte no período de 2001 a 2011, identificando a escala de prioridades em saúde, segundo as causas de mortalidade, sob a perspectiva da heterogeneidade desse grupo, considerando-se as duas faixas etárias limítrofes: 60 a 69 anos (idosos mais jovens) e de 80 anos ou mais de idade (idosos longevos).

\section{MÉTODOS}

Trata-se de estudo do tipo ecológico, sendo a unidade de observação e análise o estado do Rio Grande do Norte, localizado na Região Nordeste e com um total de 342.890 indivíduos com 60 anos ou mais de idade, o que representa $10,82 \%$ do total da população, segundo o Censo Demográfico realizado pelo Instituto Brasileiro de Geografia e Estatística (IBGE) em 2010. ${ }^{5}$

Para o estudo, foi analisado o universo de óbitos entre os anos de 2001 a 2011 no Rio
Grande do Norte, referentes aos dois extremos da faixa etária correspondente à população idosa: 60 a 69 anos (idosos jovens) e 80 anos ou mais de idade (idosos longevos), com vistas a observar a heterogeneidade presente nessa população. Quanto aos idosos de 70 a 79 anos, optou-se por não incluí-los devido à confusão de características presentes neste grupo, apresentando semelhanças com os idosos mais jovens e longevos.

As variáveis do estudo são dados agregados coletados no nível do estado do Rio Grande do Norte e correspondem aos óbitos por causas específicas, segundo local de residência. Para tanto, as Causas Básicas de Óbito foram consideradas agrupadas de acordo com os Capítulos CID-10ª Revisão. As mesmas foram medidas em função da Mortalidade Proporcional (MP), que permite identificar mais claramente as principais causas de óbito e orienta a definição da escala de prioridades em saúde.

Para o cálculo da MP, faz-se a razão do número de óbitos devido a uma causa y e a relação total de óbitos na mesma área e período, segundo a expressão da figura $1 .^{10}$ Tais informações foram obtidas através do Sistema de Informações sobre Mortalidade (SIM), capturadas no sítio do Departamento de Informática do SUS (DATASUS) e oriundas da Secretaria de Vigilância em Saúde (SVS) do Ministério da Saúde.

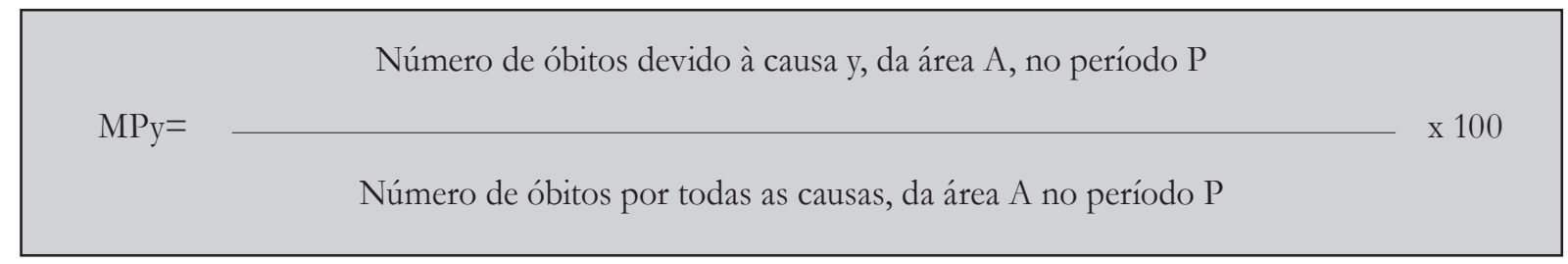

Fonte: Adaptado de Medronho et al. ${ }^{10}$

Figura 1. Cálculo da mortalidade proporcional segundo a causa do óbito. Natal-RN, 2014 


\section{RESULTADOS}

No período em questão, foram registrados 163.896 óbitos no Rio Grande do Norte, dentre os quais 21.813 ocorreram em idosos de 60 a 69 anos e 50.637 em idosos de 80 anos ou mais de idade, o que corresponde, respectivamente, a 13,31\% e $30,9 \%$ do total de óbitos para o estado. A respeito da caracterização da população estudada quanto aos aspectos sociodemográficos, os idosos mais jovens eram do sexo masculino em sua maioria $(56,5 \%)$, ao passo que no grupo de idosos longevos houve discreto predomínio de mulheres $(51,6 \%)$

Mais de $70 \%$ dos indivíduos das duas faixas etárias pertenciam à raça parda e branca, sendo a primeira mais expressiva entre os idosos mais jovens (38,6\%), ao passo que os indivíduos da raça branca atingiram maior longevidade $(47,6 \%$ do grupo de longevos). Quanto ao estado civil, $55,7 \%$ dos idosos mais jovens eram casados. Tal característica tem sua importância diminuída entre os longevos, destacando-se os indivíduos viúvos $(38,4 \%)$, sendo a proporção de solteiros semelhante nos dois grupos (cerca de 21\%).

A escolaridade de $67,9 \%$ do grupo de 60 a 69 anos não ultrapassou três anos de estudo em relação aos dados válidos, sendo 37,2\% desses sem nenhum ano de estudo. Tal situação é ainda mais grave no grupo de 80 anos ou mais, com 59,5\% dos indivíduos sem nenhum ano de estudo e $23,7 \%$ com um a três anos. Além da baixa escolaridade, observa-se a falha no preenchimento desse item, com perda de informação superior a 30\%. A respeito do local de ocorrência, 66,25\% dos óbitos em idosos mais jovens e $49,9 \%$ em longevos se deram em hospital, observando-se também alta proporção dos óbitos em domicílio no último grupo (47,5\%).

No que se refere ao perfil de mortalidade da população investigada, a figura 2 apresenta a proporção de óbitos dos capítulos mais prevalentes no período de 2001 a 2011 para os dois grupos de idade em questão.

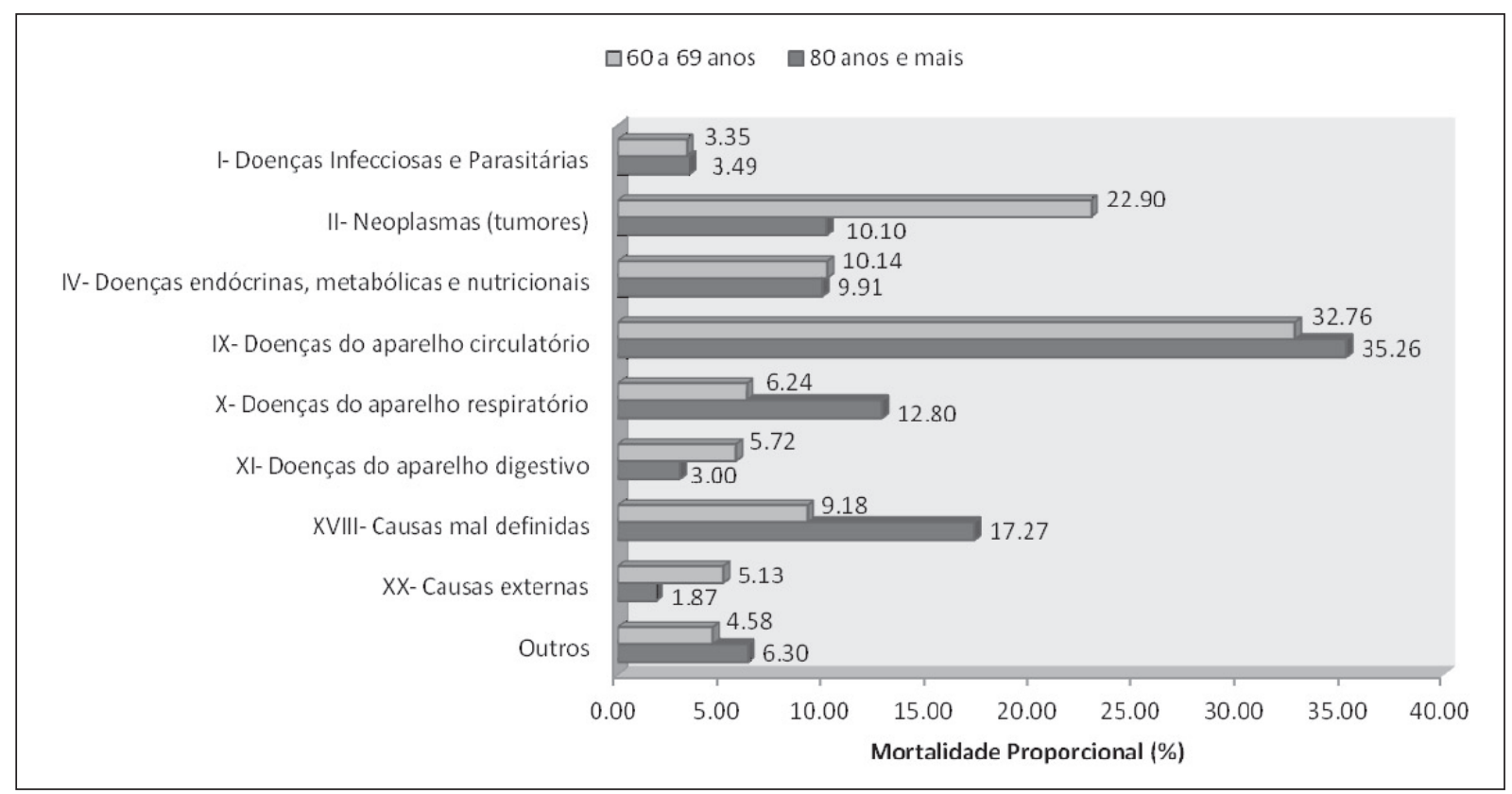

Figura 2. Mortalidade proporcional segundo capítulo CID-10 em idosos de 60 a 69 anos e 80 anos ou mais que faleceram no período de 2001 a 2011 no Estado do Rio Grande do Norte. Natal-RN, 2014. 
As doenças do aparelho circulatório são a principal causa de morte para as duas faixas etárias em questão, sendo ainda mais importantes entre os idosos longevos. Já em relação ao capítulo com a segunda maior carga de mortalidade, encontram-se diferenças expressivas entre as duas faixas etárias. Para os idosos mais jovens, o mesmo corresponde aos neoplasmas e representa um quinto do total de óbitos, ao passo que, entre os longevos, esta causa ocupa a quarta posição e causou o óbito de um décimo da população estudada.

Ainda a respeito dosidosos longevos, a segunda causa de óbito mais prevalente corresponde às mal-definidas, com uma diferença de oito pontos percentuais acima da proporção em idosos mais jovens, nas quais ocupa a quarta posição. Tal resultado reflete uma qualidade inferior da informação para os indivíduos com mais de 80 anos de idade devido à maior dificuldade de determinar a causa de óbito.

Os idosos mais jovens e os longevos apresentam importante diferença também em relação às doenças respiratórias, doenças do aparelho digestivo e causas externas. Sobre a primeira causa, observa-se que os longevos têm uma proporção duas vezes maior que os idosos mais jovens, enquanto estes possuem uma proporção cerca de duas e três vezes maior de óbitos por doenças do aparelho digestivo e causas externas, respectivamente.

\section{DISCUSSÃO}

O presente estudo apresenta achados em consonância com o perfil epidemiológico da população mundial, caracterizado pela predominância das doenças do aparelho circulatório. ${ }^{11}$ Apesar de constituir a principal causa de óbito, evidências apontam para um declínio da sua importância no Brasil, bem como em regiões desenvolvidas. Tal decréscimo pode ser atribuído aos avanços terapêuticos e tecnológicos do cuidado médico, bem como ao controle de fatores de risco. No entanto, o trabalho de Mansur et al. ${ }^{12}$ mostrou que a tendência de óbitos por doenças cardiovasculares nas regiões Nordeste e Centro-Oeste brasileiras apresentaram direção oposta, com aumento do risco de morte.

Segundo o estudo, ${ }^{12}$ o incremento da mortalidade nessas regiões pode ter sido influenciado "1) pela melhora no diagnóstico da causa de morte; 2) resultado de um aumento na urbanização; 3) piora das condições socioeconômicas dessa região; 4) baixa escolaridade; e 5) outros, ainda discutíveis, que incluem desde peso ao nascimento, a aspectos nutricionais, culturais e comportamentais". Portanto, melhorias nas condições socioeconômicas e de acesso aos serviços de saúde podem reverter essa tendência, especialmente se o diagnóstico e tratamento da hipertensão arterial, importante determinante da morbidade e mortalidade por doenças cardiovasculares, for favorecido, o que resulta em significativo retorno social com baixo custo econômico.

Além das doenças do aparelho circulatório, destacam-se as neoplasias entre os dados de mortalidade de idosos, especialmente da mortalidade precoce. Juntas, tais causas foram responsáveis por mais de $55 \%$ e $45 \%$ dos óbitos de indivíduos de 60 a 69 e 80 anos ou mais do presente estudo, respectivamente. Ao contrário das primeiras, as neoplasias têm apresentado aumento gradativo de suas taxas de mortalidade, o que pode ser explicado pela maior exposição a fatores cancerígenos. Como resultado de um processo de industrialização cada vez mais evoluído e dos atuais padrões de vida adotados em relação ao trabalho, nutrição e consumo em geral, os indivíduos se expõem a agentes químicos, físicos e biológicos cada vez mais agressivos. ${ }^{13}$

Aliada ao acúmulo de tais fatores de risco, a menor eficácia dos mecanismos de reparação celular aumenta consideravelmente a incidência 
de câncer com o avançar da idade. Portanto, a crescente importância das neoplasias se relaciona ao envelhecimento populacional, sendo as neoplasias de mama, pâncreas, cérebro, rins e próstata, as mais envolvidas com o processo de transição demográfica e epidemiológica. ${ }^{11}$ Ademais, o indivíduo idoso apresenta grandes dificuldades no tratamento do câncer, pois as intervenções médicas, na sua maioria, não são testadas em pesquisas para esse grupo e o diagnóstico muitas vezes é feito em estágios avançados da doença, devido a uma maior restrição ao encaminhamento para serviços especializados, se comparado aos adultos e jovens. ${ }^{14}$

Nesse sentido, os serviços públicos devem estar estruturados para atender a essa crescente demanda, garantindo o acesso e atendimento de qualidade desde a detecção até o tratamento da doença, bem como investindo em ações preventivas. Mesmo que a doença de base não possa ser curada, o câncer tem diversas patologias associadas passíveis de intervenção clínica, garantindo a melhor qualidade de vida possível para os pacientes e suas famílias por meio do cuidado paliativo. Para tanto, devemse considerar também as complexidades das neoplasias, que não constituem entidade nosológica única, mas um grupo de doenças com diferenças a respeito da etiologia, fatores de risco, localização, patogenicidade e tipo de célula envolvida. Desta forma, existem possibilidades terapêuticas e preventivas distintas que, dada a grande variabilidade de incidência e mortalidade nas regiões geográficas, devem ser planejadas de acordo com as especificidades da doença. ${ }^{14,15}$

Aliadas às doenças cardiovasculares e neoplásicas, as doenças respiratórias fazem parte da hierarquia das principais causas de mortalidade de idosos no Brasil. ${ }^{16}$ No presente estudo, estas representaram a segunda maior causa de óbito entre os longevos, se excluídas as causas maldefinidas, porém são menos importantes para os idosos mais jovens, entre os quais ocupam a quarta posição. A alta magnitude das taxas de mortalidade encontrada entre os idosos de 80 anos ou mais de idade também foi verificada no estudo de Campagna et al., ${ }^{17}$ o que pode estar associado à maior susceptibilidade fisiológica e imunológica com o avançar da idade.

Além disso, o alto percentual de óbitos por doenças respiratórias entre os longevos pode estar relacionado à maior frequência de acamados. Os indivíduos nesta condição podem desenvolver complicações relacionadas à fraqueza muscular diafragmática e intercostal, dificuldade em eliminar secreção, tosse menos eficaz e diminuição da função ciliar, resultando em acúmulo de secreção, predispondo-os assim a atelectasia e infecções respiratórias. ${ }^{18}$

Uma das principais estratégias adotadas para o controle das doenças respiratórias se refere à imunização com a vacina contra o vírus Influenza, que contribui consideravelmente para a redução da mortalidade e dos gastos com internações e tratamentos. ${ }^{17}$ No Brasil, a vacina contra a gripe é direcionada aos idosos e portadores de doenças crônicas, grupos de maior risco de complicações pelo vírus Influen₹a, atingindo cobertura de mais de $70 \%$ em todo o território e já apresentando evidências da sua efetividade. ${ }^{19}$ Todavia, são necessárias adaptações do calendário de vacinação de acordo com as variações regiões de sazonalidade, para que a campanha de vacinação obtenha resultados ainda mais positivos, além de se considerar a circulação de outros patógenos de grande importância clínica e epidemiológica para as doenças do aparelho respiratório. ${ }^{20}$

No presente estudo, observou-se deficiência no preenchimento da declaração de óbito em relação à determinação da causa básica, que, segundo critério adotado por Paes, ${ }^{21}$ obteve qualidade satisfatória dos dados (proporção de causas mal-definidas inferior a 10\%) para os idosos de 60 a 69 anos, porém regular (17,3\% de causas desconhecidas) para aqueles de 80 anos ou mais. Tendo em vista que parcela significativa dos óbitos entre os longevos se deu no ambiente domiciliar, o alto índice de causas mal-definidas pode ser explicado pela ausência de assistência médica. 
Para facilitar a identificação das causas específicas dos óbitos nos casos de cuidado domiciliar, recomenda-se que a atenção básica em saúde acompanhe e faça o registro prévio do quadro de saúde dos pacientes de sua comunidade. $^{22}$ Nesse sentido, destaca-se o papel da Caderneta de Saúde da Pessoa Idosa, que permite identificar sinais de risco para o adoecimento orgânico por meio de levantamento periódico realizado por profissionais da atenção básica. ${ }^{23}$ Deste modo, tal instrumento auxilia na prevenção de doenças, na avaliação constante das condições de saúde e, consequentemente, na determinação da causa básica de morte.

A alta proporção de óbitos por causas maldefinidas prejudica a análise da distribuição da mortalidade segundo causas, pois pode alterar a ordem real de prioridades baseada no impacto das diferentes causas, interferindo na alocação racional de recursos de saúde. ${ }^{24}$ Segundo Fajardo, Aerts \& Bassanesi, ${ }^{25}$ além das características do falecido e da causa do óbito, falhas na acurácia da declaração de óbito podem ser influenciadas por características relacionadas ao atestante. Sendo assim, é possível elevar a qualidade da informação.

Para tanto, faz-se necessário que o preparo pelas escolas médicas aborde mais profundamente a finalidade e ensino do preenchimento da declaração de óbito, das causas de morte e de definições e usos das estatísticas de mortalidade. Projetos de educação continuada sobre o tema devem ser incentivados pelo Conselho Federal de Medicina, instituições de ensino e de saúde e Ministério da Saúde, cabendo a este também a revisão e aprimoramento do formulário. Ademais, as secretarias municipais de Saúde podem contribuir estabelecendo um sistema de vigilância sobre a qualidade da informação fornecida pelos médicos e codificadores. ${ }^{26-28}$

Para os dois grupos de idade investigados, as doenças endócrinas, metabólicas e nutricionais apresentaram importância semelhante como causa de mortalidade, com percentual de aproximadamente 10\%. Entre essas doenças, a literatura aponta o diabetes mellitus como aquela que detém a maior carga de óbitos, apresentando tendência de crescimento exponencial de sua prevalência com a transição nutricional e adquirindo proporções epidêmicas em vários países. ${ }^{29}$ Segundo Sartorelli \& Franco, ${ }^{29}$ "as alterações no estilo de vida, com ênfase na alimentação e prática de atividades físicas, associadas ao aumento da esperança de vida dos brasileiros, são atualmente apontados como os principais fatores responsáveis pelo aumento da prevalência do diabetes tipo 2 observado no país". No entanto, acredita-se que há uma subestimação do diabetes mellitus como causa básica, devido ao mal preenchimento da declaração de óbito, na qual esta doença é muitas vezes erroneamente classificada como causa contribuinte. ${ }^{28,30}$

Entre as doenças endócrinas, metabólicas e nutricionais, destaca-se também a desnutrição, que surge mais como causa associada que como básica. Tal agravo representa potencial coadjuvante na determinação da ocorrência de óbitos em idosos por outras causas, pois agrava as doenças em atividade, torna maior a susceptibilidade a infecções e aumenta os riscos para outras morbidades. Nesse sentido, para o estudo da desnutrição, considerar apenas a causa básica gera perda de informações, podendo sua magnitude ser subdimensionada cerca de cinco vezes. A real magnitude da desnutrição é mais bem captada no estudo das causas múltiplas de morte, que considera todas as causas (básica, consequenciais e contribuintes), mencionadas na declaração de óbito. ${ }^{31}$

As características correspondentes a este capítulo da CID-10 evidenciam a limitação em se utilizar apenas a causa básica para o estudo da mortalidade, especialmente entre o grupo de idosos, no qual é frequente a presença de múltiplas morbidades. Considerar todas as menções de causa declaradas na declaração de óbito é uma alternativa para retratar um perfil de mortalidade mais próximo da realidade e, portanto, deve-se considerar o aprimoramento do Sistema de Informações sobre Mortalidade no sentido de divulgar as doenças associadas à causa básica. ${ }^{30,32}$ 
As causas externas, as doenças do aparelho digestivo e as infecciosas e parasitárias apresentaram menor impacto no padrão de mortalidade da população estudada, com proporção inferior a $6 \%$ dos óbitos para cada grupo. No que se refere às duas primeiras causas, observa-se que produziram maior impacto entre os dados de mortalidade dos idosos mais jovens, o que pode ter sido influenciado pela predominância de indivíduos do sexo masculino na faixa etária em questão. Segundo Laurenti, Mello Jorge \& Gotlieb, ${ }^{32}$ há acentuada sobremortalidade masculina para essas duas causas, sendo as doenças do aparelho digestivo destacadamente representadas pela cirrose hepática (informada ou não com associação ao alcoolismo) e causas externas por homicídios e acidentes de transporte. Ademais, acredita-se que os indivíduos longevos estão menos expostos aos fatores de risco relacionados a tais causas específicas.

Apesar da pequena representatividade das doenças do aparelho digestivo no total de óbitos, também observada em todo o território nacional, o estudo de Vasconcelos ${ }^{33}$ revelou crescimento de mais de $200 \%$ de suas taxas num período de dez anos, o que pode ser consequência dos novos procedimentos de coleta, apuração e tratamento das informações sobre causas de morte. Já quando se consideram os dados de morbidade, as doenças do aparelho digestivo são apontadas como uma das principais causas de internação tanto em idosos, quanto na população em geral. ${ }^{34}$

Da mesma forma, as reduzidas proporções de óbitos por doenças infecciosas e parasitárias (pouco mais de 3\% para os dois grupos de idade) não acompanham o perfil de morbidade da população, que ainda apresenta elevadas taxas de tuberculose, hanseníase, malária e leishmaniose. ${ }^{35}$ Nesse contexto, vale destacar que o presente estudo possui a limitação de refletir parcialmente o perfil epidemiológico da população, visto que as enfermidades de menor letalidade não são bem representadas pelos indicadores de mortalidade. Acrescentase a isso, a alta prevalência de doenças crônicas, associadas à presença de múltiplas morbidades, que evidencia a necessidade de considerar as estatísticas de mortalidade e morbidade simultaneamente para a descrição mais fidedigna da situação de saúde..$^{28,35}$

\section{CONCLUSÃO}

No presente estudo, é possível observar a heterogeneidade da faixa etária idosa quanto ao padrão de mortalidade. Tendo em vista o impacto desse grupo sobre o perfil epidemiológico da população, faz-se necessário considerar as distintas escalas de prioridades no planejamento, ações e estratégias em saúde.

A principal causa de óbito, as doenças do aparelho circulatório, é comum aos dois grupos de idade, porém a maior parte das causas apresentam importâncias diferentes entre os mesmos. As neoplasias, doenças do aparelho digestivo e causas externas detêm maior necessidade de atenção por parte das políticas públicas para os idosos mais jovens, ao passo que as causas mal-definidas e as doenças do aparelho respiratório são mais significativas para os idosos de idade mais avançada.

Ademais, destaca-se a precariedade das informações no que diz respeito à determinação da causa básica dos óbitos, sendo a qualidade dos dados considerada regular para os indivíduos de 80 anos de idade ou mais, o que limita o delineamento de um perfil epidemiológico mais próximo da realidade. 


\section{REFERÊNCIAS}

1. Silva VL, Albuquerque MFPM, Cesse EAP, Luna CF. Perfil de mortalidade do idoso: análise da evolução temporal em uma capital do Nordeste brasileiro de 1996 a 2007. Rev Bras Geriatr Gerontol 2012;15(3):433-41.

2. Ishitani LH, França E. Uso das causas múltiplas de morte em saúde pública. Inf Epidemiol SUS 2001;10(4):163-75.

3. Araujo JD. Polarização epidemiológica no Brasil. Epidemiol Serv Saúde 2012;21(4):533-8.

4. Omran AR. The epidemiologic transition: a theory of the epidemiology of population change. Milbank Meml Q 1971;49(4):509-38.

5. Brasil. Ministério da Saúde. Portal da Saúde. Datasus [Internet]. Brasília, DF: c2008- . Informações de Saúde; [acesso em 10 Jan 2012]. Disponível em: www2.datasus.gov.br.

6. Gotlieb MGV, Schwanke CHA, Gomes I, Cruz IBM. Envelhecimento e longevidade no Rio Grande do Sul: um perfil histórico, étnico e de morbi-mortalidade dos idosos. Rev Bras Geriatr Gerontol 2011;14(2):365-80.

7. Luz EP, Dallepiane LB, Kirchner RM, Silva LAA, Silva FP, Kohler J, et al. Perfil sociodemográfico e de hábitos de vida da população idosa de um município da região norte do Rio Grande do Sul, Brasil. Rev Bras Geriatr Gerontol 2014;17(2):303-14.

8. Rosset I, Pedrazzi EC, Roriz-Crus M, Morais EP, Rodrigues RAP. Tendências dos estudos com idosos mais velhos na comunidade: uma revisão sistemática (inter)nacional. Rev Esc Enferm USP 2011;45(1):264-71.

9. Papeleo Netto M. Fisiologia do envelhecimento: aspectos fundamentais. In: Papeleo Netto M, Brito FC. Urgências em geriatria. São Paulo: Atheneu; 2001. p. 1-22.

10. Medronho RA, Bloch KV, Luiz RR, Werneck GL. Epidemiologia. 2a ed. Rio de Janeiro: Atheneu; 2009.

11. Lozano R, Naghavi M, Foreman K, Lim S, Shibuya K, Aboyans V, et al. Global and regional mortality from 235 causes of death for 20 age groups in 1990 and 2010: a systematic analysis for the global burden of disease study 2010. Lancet 2012;380(9859):2095-128.

12. Mansur AP, Souza MFM, Timerman A, Avakian SD, ALdrighi JM, Ramires JAF. Tendência do risco de morte por doenças circulatórias, cerebrovasculares e isquêmicas do coração em treze Estados do Brasil, de 1980 a 1998. Arq Bras Cardiol 2006;87(5):641-8.
13. Brasil. Instituto Nacional do Câncer. Ações de enfermagem para o controle do câncer: uma proposta de integração ensino-serviço. $3^{\mathrm{a}}$ ed. Rio de Janeiro: INCA; 2008.

14. Mathias TAF, Mello Jorge MHP. Evolução da mortalidade por neoplasias em idosos em município do estado do Paraná, 1979-1998. Ciênc Cuid Saúde 2006;5(1):57-64.

15. Hanna SA, Marta GN, Santos FS. O médico frente a novidades no tratamento do câncer: quando parar? Rev Assoc Med Bras 2011;57(5):588-93.

16. Alves DSB. Análise espacial da mortalidade de idosos por doenças crônicas no município do Rio de Janeiro [dissertação]. Rio de Janeiro: Escola Nacional de Saúde Pública Sergio Arouca; 2013.

17. Campagna AS, Dourado I, Duarte EC, Daufenbach LZ. Mortalidade por causas relacionadas à influenza em idosos no Brasil, 1992 a 2005. Epidemiol Serv Saúde 2009;18(3):209-18.

18. Fonseca HLA. Caracterização dos principais problemas de saúde do idoso acamado, e desafio para a equipe de saúde da família no acompanhamento destes [trabalho de conclusão de curso]. Belo Horizonte: Universidade Federal de Minas Gerais; 2010.

19. Daufenbach LZ, Carmo EH, Duarte EC, Campagna AS, Teles CAS. Morbidade hospitalar por causas relacionadas à influenza em idosos no Brasil, 1992 a 2006. Epidemiol Serv Saúde 2009;18(1):29-44.

20. Gomes AA, Nunes MAP, Oliveira CCC, Lima SO. Doenças respiratórias por influenza e causas associadas em idosos de um município do Nordeste brasileiro. Cad Saúde Pública 2013;29(1):117-22.

21. Paes NA. Qualidade das estatísticas de óbitos por causas desconhecidas dos Estados brasileiros. Rev Saúde Pública 2007;41(3):436-45.

22. Mendes JDV. Mortalidade proporcional por causas mal definidas nas regiões do estado de São Paulo. GAIS Inf 2012;4(12):1-9.

23. Brasil. Ministério da Saúde, Secretaria de Atenção à Saúde, Departamento de Ações Programáticas e Estratégicas. Caderneta de saúde da pessoa idosa : manual de preenchimento. Brasília, DF : Ministério da Saúde; 2008. (Série A. Normas e Manuais Técnicos).

24. Mello Jorge MHP, Laurenti R, Gotlieb SL. Análise da qualidade das estatísticas vitais brasileiras: a experiência de implantação do SIM e do SINASC. Ciênc Saúde Coletiva 2007;12(3):643-54. 
25. Fajardo S, Aerts DRGC, Bassanesi SL. Acurácia da equipe do sistema de informações sobre mortalidade na seleção da causa básica do óbito em capital no Sul do Brasil. Cad Saúde Pública 2009;25(10):2218-28.

26. Silva JAC, Yamaki VN, Oliveira JPS, Teixeira RKC, Santos FAF, Hosoume VSN. Declaração de óbito, compromisso no preenchimento. Avaliação em Belém Pará, em 2010. Rev Assoc Med Bras 2013;59(4):335-40.

27. Mendonça FM, Drumond E, Cardoso AMP. Problemas no preenchimento da Declaração de Óbito: estudo exploratório. Rev Bras Estud Popul 2010;27(2):285-95.

28. Laurenti R, Mello Jorge MHP, Gotlieb SLD. Mortalidade segundo causas: considerações sobre a fidedignidade dos dados. Rev Panam Salud Publica 2008;23(5):349-56.

29. Sartorelli DS, Franco JL. Tendências do diabetes mellitus no Brasil: o papel da transição nutricional. Cad Saúde Pública 2003;19 Supl 1:S29-36.

30. Cascão AM, Costa AJL, Kale PL. Qualidade da informação sobre mortalidade numa coorte de diabéticos - estado do Rio de Janeiro, 2000 a 2003. Rev Bras Epidemiol 2012;15(1):134-42.

31. Rezende EM, Sampaio IBM, Ishitani LH, Martins EF, Vilella LCM. Mortalidade de idosos com desnutrição em Belo Horizonte, Minas Gerais, Brasil: uma análise multidimensional sob o enfoque de causas múltiplas de morte. Cad Saúde Pública 2010;26(6):1109-21.

32. Laurenti R, Mello Jorge MHP, Gotlieb SLD. Perfil epidemiológico da morbi-mortalidade masculina. Ciênc Saúde Coletiva 2005;10(1):35-46.

33. Vasconcelos AMN. Causas de morte em idosos no Brasil. In: Anais do $14^{\circ}$ Encontro Nacional de Estudos Populacionais; 20-24 set 2004; Caxambú. Minas gerais: ABEP; 2004. p. 1-11.

34. Garcia PL, Montenegro MMS, Ramalho WM. Mortalidade no Brasil: situação de 2008 evolução segundo principais grupos de causas no período de 1980 a 2008. In: Brasil. Ministério da Saúde, Secretaria de Vigilância em Saúde, Departamento de Análise de Situação de Saúde. Saúde Brasil 2009: uma análise da situação de saúde e da agenda nacional e internacional de prioridades em saúde. Brasília: Ministério da Saúde; 2010. p. 45-71.

35. Sousa-Muñoz RL, Ronconi DE, Dantas GC, Lucena DMS, Silva IBA. Impacto de multimorbidade sobre mortalidade em idosos: estudo de coorte pós-hospitalização. Rev Bras Geriatr Gerontol 2013;16(3):579-89. 